\title{
Electrochemical gradients are involved in regulating cytoskeletal patterns during epithelial morphogenesis in the Drosophila ovary
}

\author{
Isabel Weiß and Johannes Bohrmann* (1)
}

\begin{abstract}
Background: During Drosophila oogenesis, the follicular epithelium differentiates into several morphologically distinct follicle-cell populations. Characteristic bioelectrical properties make this tissue a suitable model system for studying connections between electrochemical signals and the organisation of the cytoskeleton. Recently, we have described stage-specific transcellular antero-posterior and dorso-ventral gradients of intracellular $\mathrm{pH}\left(\mathrm{pH}_{\mathrm{i}}\right)$ and membrane potential $\left(V_{\text {mem }}\right)$ depending on the asymmetrical distribution and/or activity of various ion-transport mechanisms. In the present study, we analysed the patterns of basal microfilaments (bMF) and microtubules (MT) in relation to electrochemical signals.
\end{abstract}

Results: The bMF- and MT-patterns in developmental stages 8 to 12 were visualised using labelled phalloidin and an antibody against acetylated a-tubulin as well as follicle-cell specific expression of GFP-actin and GFP-a-tubulin.

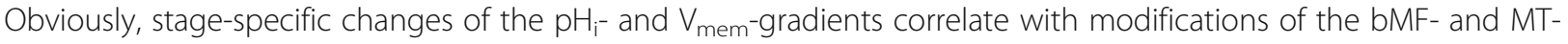
organisation. In order to test whether cytoskeletal modifications depend directly on bioelectrical changes, we used inhibitors of ion-transport mechanisms that have previously been shown to modify $\mathrm{pH}_{i}$ and $\mathrm{V}_{\text {mem }}$ as well as the respective gradients. We inhibited, in stage $10 \mathrm{~b}, \mathrm{Na}^{+} / \mathrm{H}^{+}$-exchangers and $\mathrm{Na}^{+}$-channels with amiloride, V-ATPases with bafilomycin, ATP-sensitive $\mathrm{K}^{+}$-channels with glibenclamide, voltage-dependent $\mathrm{L}$-type $\mathrm{Ca}^{2+}$-channels with verapamil, $\mathrm{Cl}^{-}$-channels with 9-anthroic acid and $\mathrm{Na}^{+} / \mathrm{K}^{+} / 2 \mathrm{Cl}^{-}$-cotransporters with furosemide, respectively. The correlations between $\mathrm{pH}_{\mathrm{i}}, \mathrm{V}_{\text {mem }}, \mathrm{bMF}$ and $\mathrm{MT}$ observed in different follicle-cell types are in line with the correlations resulting from the inhibition experiments. While relative alkalisation and/or hyperpolarisation stabilised the parallel transversal alignment of bMF, acidification led to increasing disorder and to condensations of bMF. On the other hand, relative acidification as well as hyperpolarisation stabilised the longitudinal orientation of MT, whereas alkalisation led to loss of this arrangement and to partial disintegration of MT.

Conclusions: We conclude that the $\mathrm{pH}_{\mathrm{i}}$ - and $\mathrm{V}_{\text {mem }}$-changes induced by inhibitors of ion-transport mechanisms simulate bioelectrical changes occurring naturally and leading to the cytoskeletal changes observed during differentiation of the follicle-cell epithelium. Therefore, gradual modifications of electrochemical signals can serve as physiological means to regulate cell and tissue architecture by modifying cytoskeletal patterns.

Keywords: Drosophila melanogaster, Bioelectricity, Intracellular pH, Membrane potential, Cell polarity, Pattern formation, Ion pump, Ion channel, Microfilament, Microtubule

\footnotetext{
* Correspondence: bohrmann@bio2.rwth-aachen.de

Institut für Biologie II, Abt. Zoologie und Humanbiologie, RWTH Aachen

University, Worringerweg 3, 52056 Aachen, Germany
}

(c) The Author(s). 2019 Open Access This article is distributed under the terms of the Creative Commons Attribution 4.0 International License (http://creativecommons.org/licenses/by/4.0/), which permits unrestricted use, distribution, and reproduction in any medium, provided you give appropriate credit to the original author(s) and the source, provide a link to the Creative Commons license, and indicate if changes were made. The Creative Commons Public Domain Dedication waiver (http://creativecommons.org/publicdomain/zero/1.0/) applies to the data made available in this article, unless otherwise stated. 


\section{Background}

Localised ion fluxes, gradients of ion concentrations, of intracellular $\mathrm{pH}\left(\mathrm{pH}_{\mathrm{i}}\right)$ and of membrane potentials $\left(\mathrm{V}_{\text {mem }}\right)$ are involved, as fast and wide-ranging signals, in various developmental and regenerative processes [1-6]. Gradual changes of bioelectrical properties mediate diverse cellular events, e.g. proliferation [7], migration [8] and differentiation [9-12]. Establishing electrochemical gradients within single cells or whole tissues requires asymmetrically distributed or activated ion-transport mechanisms [13-16] as well as gap junctions [17-21].

Electrochemical signals are transduced, perceived and translated into cellular responses by $\mathrm{pH}_{\mathrm{i}^{-}}$or $\mathrm{V}_{\text {mem-sensi- }}$ tive ion-channels, phosphatases, transporters of signalling molecules or other proteins, like elements of the cytoskeleton $[2,22]$. Influences of $\mathrm{pH}_{\mathrm{i}}$ on actin selfassembly [23], on contractility of the actomyosin cytoskeleton [24] and on the activity of cross-linking proteins $[25,26]$ are known to exist. Furthermore, changes of $\mathrm{V}_{\text {mem }}$ are associated with reorganisation or stabilisation of the microfilament (MF) network [27, 28]. Besides $\mathrm{pH}_{\mathrm{i}}$-dependence of polymerisation as well as depolymerisation of microtubules (MT) [25, 29, 30], correlations between $\mathrm{V}_{\text {mem }}$-changes and an altered MT-organisation are also known [31-33].
We have found, in ovarian follicles of Drosophila melanogaster, stage-specific patterns of extracellular currents [34], gradients of $\mathrm{pH}_{\mathrm{i}}[15,16]$ and gradients of $\mathrm{V}_{\text {mem }}[15,16,35]$. It is tempting to assume that these bioelectrical phenomena, resulting mainly from the exchange of protons, potassium ions and sodium ions [3539], serve as signals to guide development. During the course of oogenesis, follicles consisting of 16 germ-line cells, i.e. 15 nurse cells (NC) and one oocyte (Oo), surrounded by a single-layered somatic follicle-cell epithelium (FCE) are passing through 14 stages (S1-14) [40] (Fig. 1). The FCE differentiates into several morphologically distinct follicle-cell (FC) populations [41-43] with characteristic cytoskeletal patterns. Therefore, the FCE is an appropriate model system for studying influences of bioelectrical signals on the cytoskeletal organisation during development. The FCE participates in establishing the embryonic axes [44-46] and in synthesising the multi-layered eggshell [43]. Polarised and parallel aligned MF-bundles (bMF) at the basal side of the FCE have long been assumed to be involved, as a molecular corset, in shaping the egg $[47,48]$. Recent studies have demonstrated the role of bMF, and also of MT, during follicle elongation, a complex process which includes a global rotation of the FCE during S5-8 [49-53].

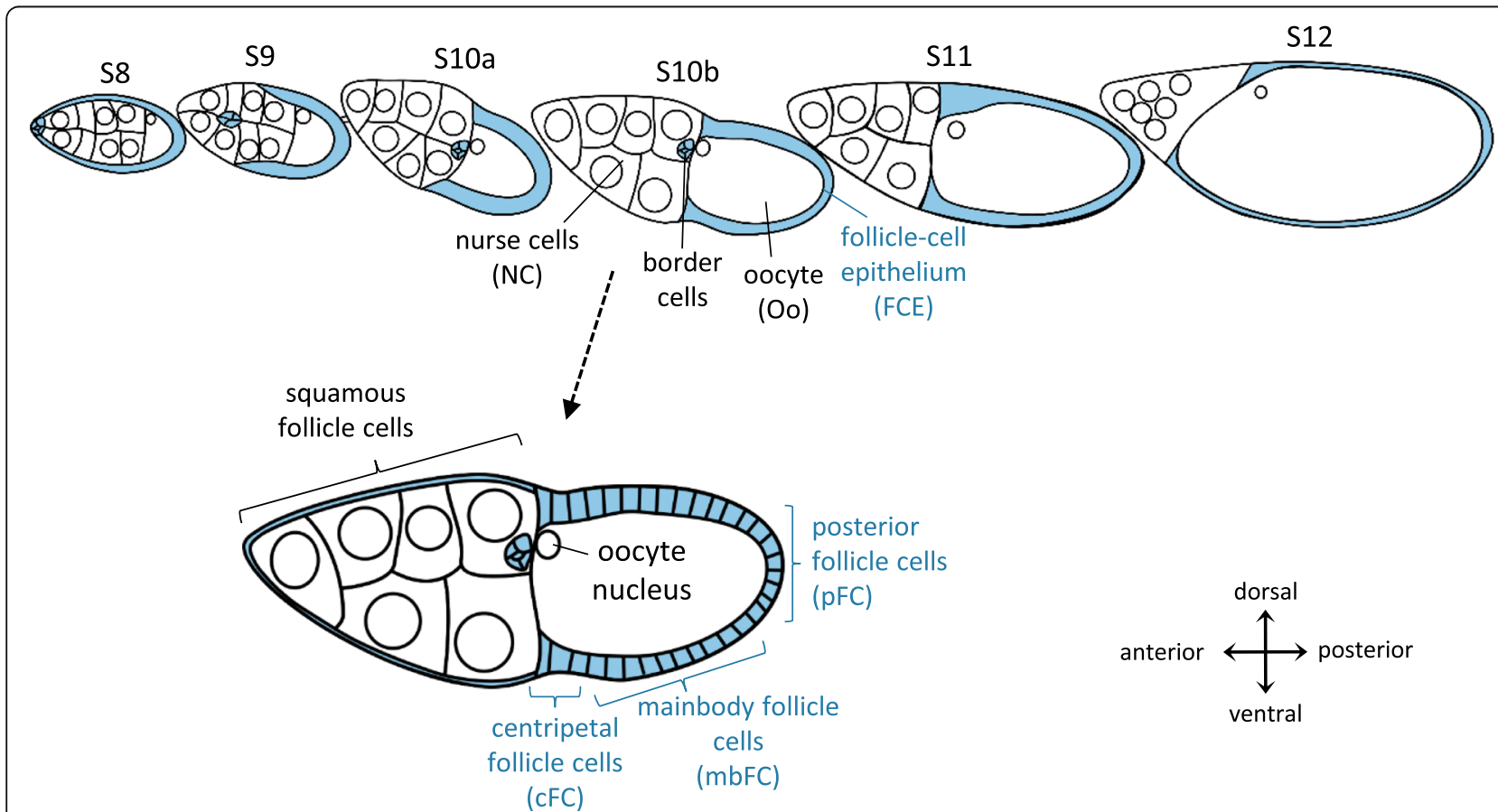

Fig. 1 Schematic drawing of the analysed stages of oogenesis. The somatic follicle-cell epithelium (FCE) that surrounds the 15 nurse cells (NC, anterior) and the oocyte (Oo, posterior) is highlighted in blue. During vitellogenic stages 8-12 (S8-12), the FCE undergoes morphological changes and differentiates into several distinct follicle-cell (FC) populations: squamous FC, surrounding the NC, border cells, centripetally migrating FC ( $\mathrm{CFC}$ ), mainbody FC (mbFC) and posterior FC ( $\mathrm{pFC}$ ), surrounding the Oo. From S10b onward, the dorsal FCE (defined by the position of the Oo nucleus) becomes thicker than the ventral FCE. Now, the Oo constitutes almost one half of the follicle's volume 


\section{a Role of ion-transport mechanisms in regulating bioelectrical properties}

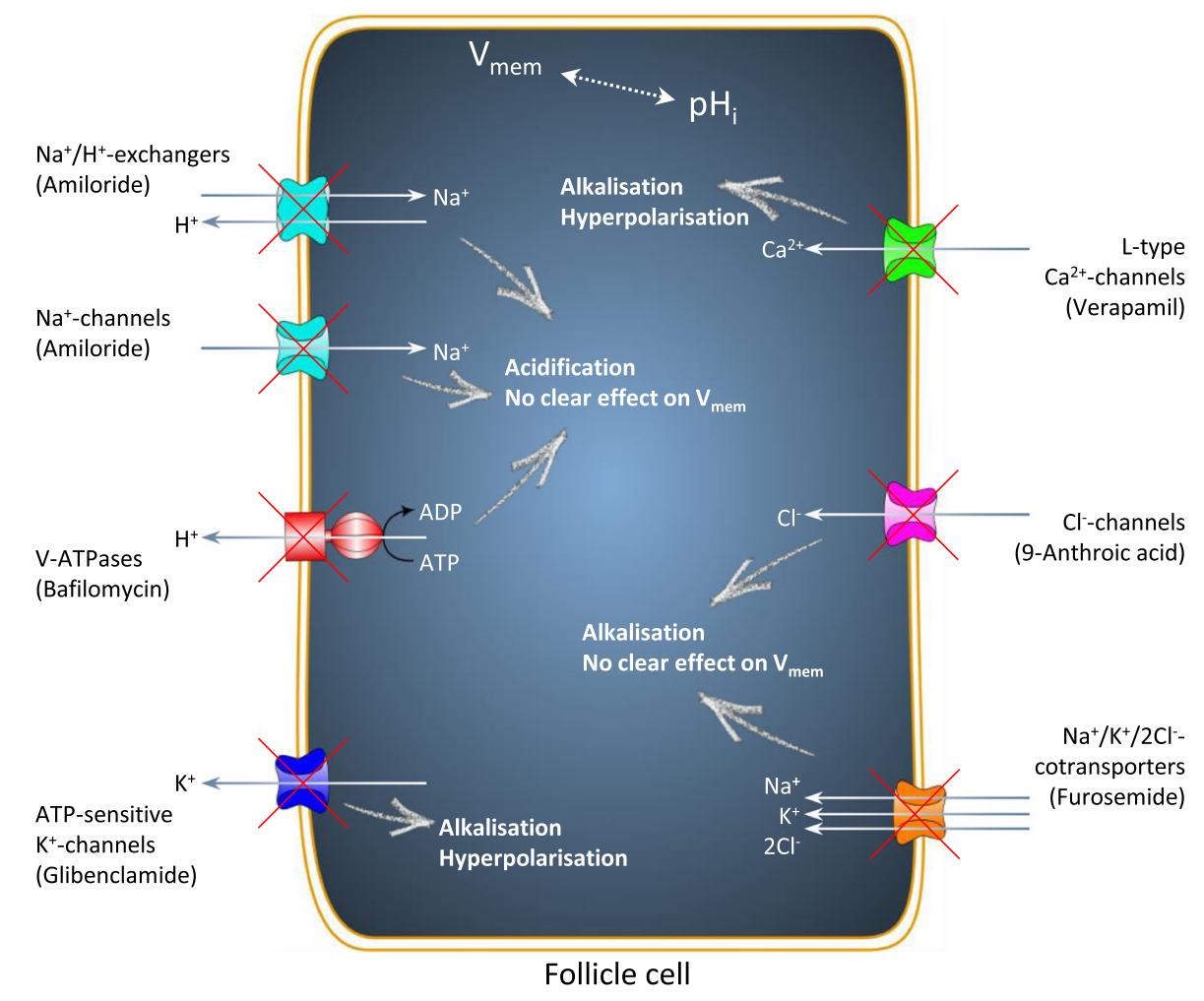

\section{b Effects of inhibitors on electrochemical gradients}
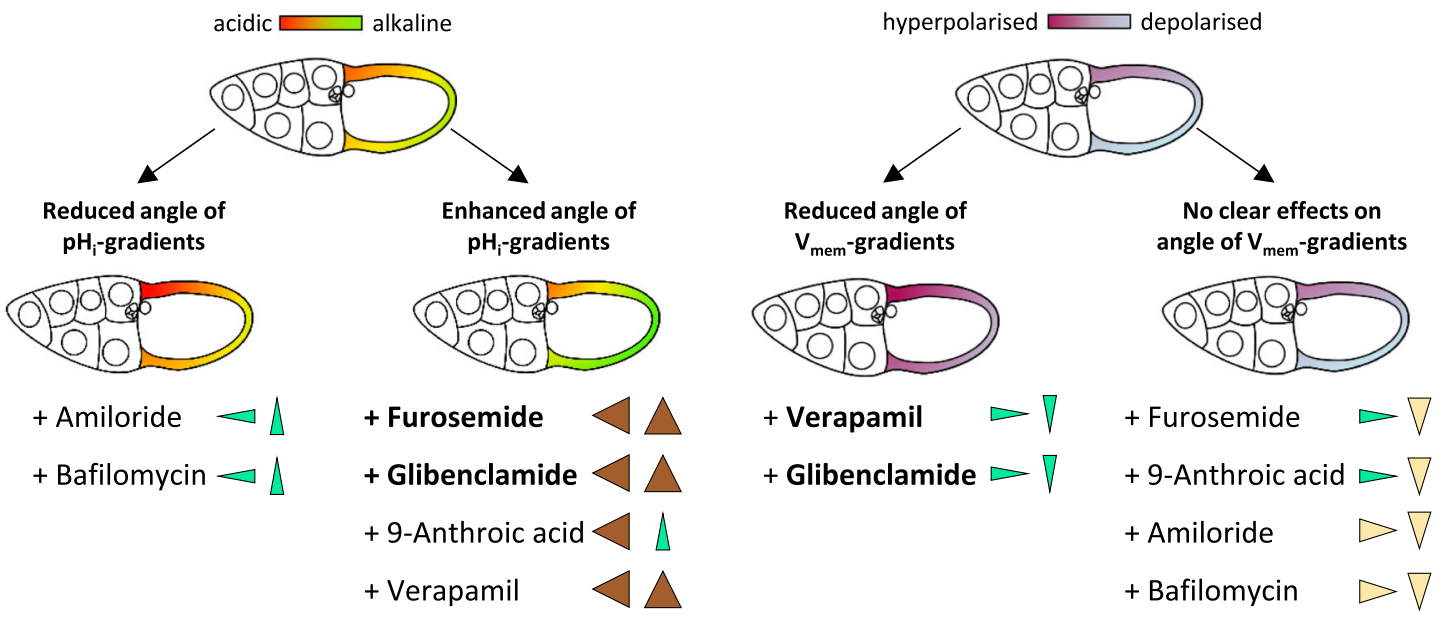

reduced angle of gradients

$<$ a-p $\mathrm{pH}_{\mathrm{i}}$-gradient (increasing $\mathrm{pH}_{\mathrm{i}}$ )

no clear effects on angle of gradients

$\triangle \mathrm{d}$ - $\mathrm{p}_{\mathrm{i}}$-gradient (increasing $\mathrm{pH}_{\mathrm{i}}$ )

enhanced angle of gradients

$\nabla$ a-p $\mathrm{V}_{\text {mem }}$-gradient (decreasing $\mathrm{V}_{\text {mem }}$ )

$\nabla$ d-v $V_{\text {mem }}$-gradient (decreasing $V_{\text {mem }}$ )

Fig. 2 (See legend on next page.) 
(See figure on previous page.)

Fig. 2 Bioelectrical properties were modified using inhibitors of ion-transport mechanisms (summarised according to [16]). a Schematic drawing of a follicle cell showing the analysed ion-transport mechanisms. $\mathrm{Na}^{+} / \mathrm{H}^{+}$-exchangers (NHE) and $\mathrm{Na}^{+}$-channels were blocked with amiloride, $\mathrm{V}$ ATPases with bafilomycin, ATP-sensitive $\mathrm{K}^{+}$-channels with glibenclamide, voltage-dependent $\mathrm{L}$-type $\mathrm{Ca}^{2+}$-channels with verapamil, $\mathrm{Cl}^{-}$-channels with 9-anthroic acid and $\mathrm{Na}^{+} / \mathrm{K}^{+} / 2 \mathrm{Cl}^{-}$-cotransporters with furosemide. Intracellular $\mathrm{pH}\left(\mathrm{pH}_{\mathrm{i}}\right)$ and membrane potential $\left(\mathrm{V}_{\text {mem }}\right)$ were analysed in living follicles using the $\mathrm{pH}$-indicator 5-CFDA,AM (5-carboxyfluorescein diacetate, acetoxymethyl ester) and the potentiometric dye $\mathrm{DiBAC}_{4}(3)$ (bis-(1,3-dibutylbarbituric acid) trimethine oxonol). $\mathrm{pH}_{\mathrm{i}}, \mathrm{V}_{\text {mem }}$ or both parameters were affected by each inhibitor [16]. b Schematic summary of the effects of inhibitors on the electrochemical gradients in the columnar FCE during S10b [16]. The antero-posterior (a-p) and dorso-ventral ( $d-v)$ $\mathrm{pH}_{\mathrm{i}}$ - and $\mathrm{V}_{\mathrm{mem}}$-gradients are visualised as colour gradients in the FCE. Triangles symbolise directions of the gradients. Increasing $\mathrm{pH}_{\mathrm{i}}$ means more alkaline, decreasing $V_{\text {mem }}$ means less hyperpolarised. The effects of inhibitors on the angles of the gradients are represented by width and colour of the triangles. While the strongest effects on $\mathrm{pH}_{i}$-gradients were generated by furosemide or glibenclamide, the strongest effects on $V_{\text {mem }}{ }^{-}$ gradients were generated by verapamil or glibenclamide (bold letters) [16]

The aim of the present study is to characterise the physiological relevance of electrochemical gradients by investigating their influence on the cytoskeletal organisation during Drosophila oogenesis. We observed stage-specific bMF- and MT-patterns in the FCE and found correlations with the stage-specific bioelectrical patterns described previously [16]. In addition, we used inhibitors of various ion-transport mechanisms, which we have recently shown to modify $\mathrm{pH}_{\mathrm{i}}$ and $\mathrm{V}_{\text {mem }}$ as well as the respective gradients during S10b (Fig. 2; [16]). We detected alterations of the bMF- and MT-patterns that result from changes in $\mathrm{pH}_{\mathrm{i}^{-}}$and $\mathrm{V}_{\mathrm{mem}}$-gradients and discuss the potential mechanisms.

\section{Results}

\section{Stage-specific patterns of basal microfilaments}

We analysed in detail, during vitellogenic stages S812 , the bMF-organisation in the cuboidal and columnar FCE (Fig. 3) and detected, despite of some variation, characteristic stage-specific patterns (Fig. 4). The bMF-bundles in the cuboidal FCE of S8 are highly polarised perpendicular to the antero-posterior (a-p) axis of the follicle (circumferential organisation). This parallel alignment, both within individual FC and in relation to neighbouring FC, disappears in part during S9. In the flattening $\mathrm{FC}$ (the prospective $\mathrm{cFC}$ ) near the border between $\mathrm{NC}$ and Oo, condensations of bMF become obvious. The bMF-bundles in the remaining columnar FCE surrounding the Oo retain their parallel alignment within individual FC, but they become more disordered relative to neighbouring FC. During S10a, the bMF-bundles in cFC are again aligned in parallel and oriented circumferentially. Subsequent morphological changes during S10b, like thickening of the dorsal FCE and elongation of inwardly migrating cFC, are accompanied by bMFcondensations that first appear in dorsal $\mathrm{cFC}$ and spread out over mbFC to pFC during S11. In S11, a peculiar bMF-organisation showing crescent- or fanshaped condensations becomes obvious, wheras during S12, a new pattern of dense parallel bMF oriented circumferentially appears (Fig. 4).

\section{Stage-specific patterns of microtubules}

A detailed analysis of the MT-organisation also revealed characteristic stage-specific patterns during S8-12 (Fig. 5). In S8, similar to bMF, the preferred orientation of MT in the cuboidal FC is perpendicular to the a-p axis of the follicle. From S9 onward, diffuse MT surround the FC nuclei in a basket-like arrangement. In the flattening $\mathrm{CFC}$, a longitudinal orientation of MT along the a-p axis first becomes obvious. During S10a-12, this longitudinal pattern continuously spreads out to mbFC and pFC. During S9-12, the MT of squamous FC covering the $\mathrm{NC}$ are organised in typical web-like structures enclosing the nuclei (Fig. 5).
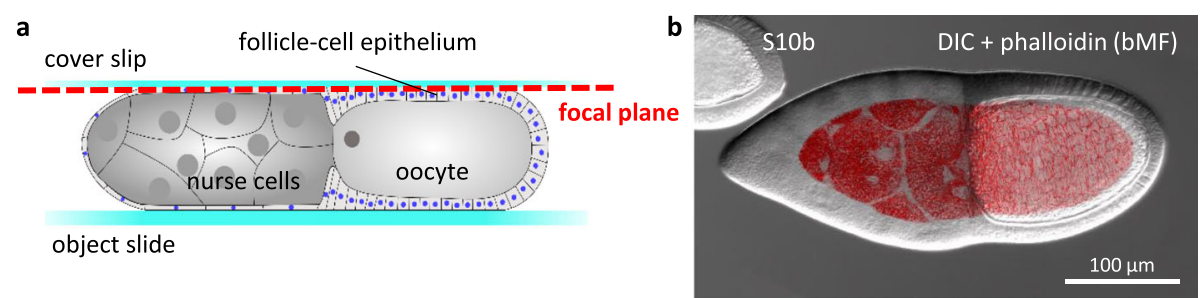

Fig. 3 Optical sectioning using structured-illumination microscopy. a Schematic drawing of a S10b-follicle placed between an object slide and a cover slip. The focal plane of tangential optical sections to analyse basal microfilaments (bMF) and microtubules (MT) in the FCE is shown as dashed red line. b Overlay of a differential interference-contrast (DIC) image and a fluorescent-phalloidin image showing the analysed area of bMF in the FCE (and, in addition, in the NC) in S10b 

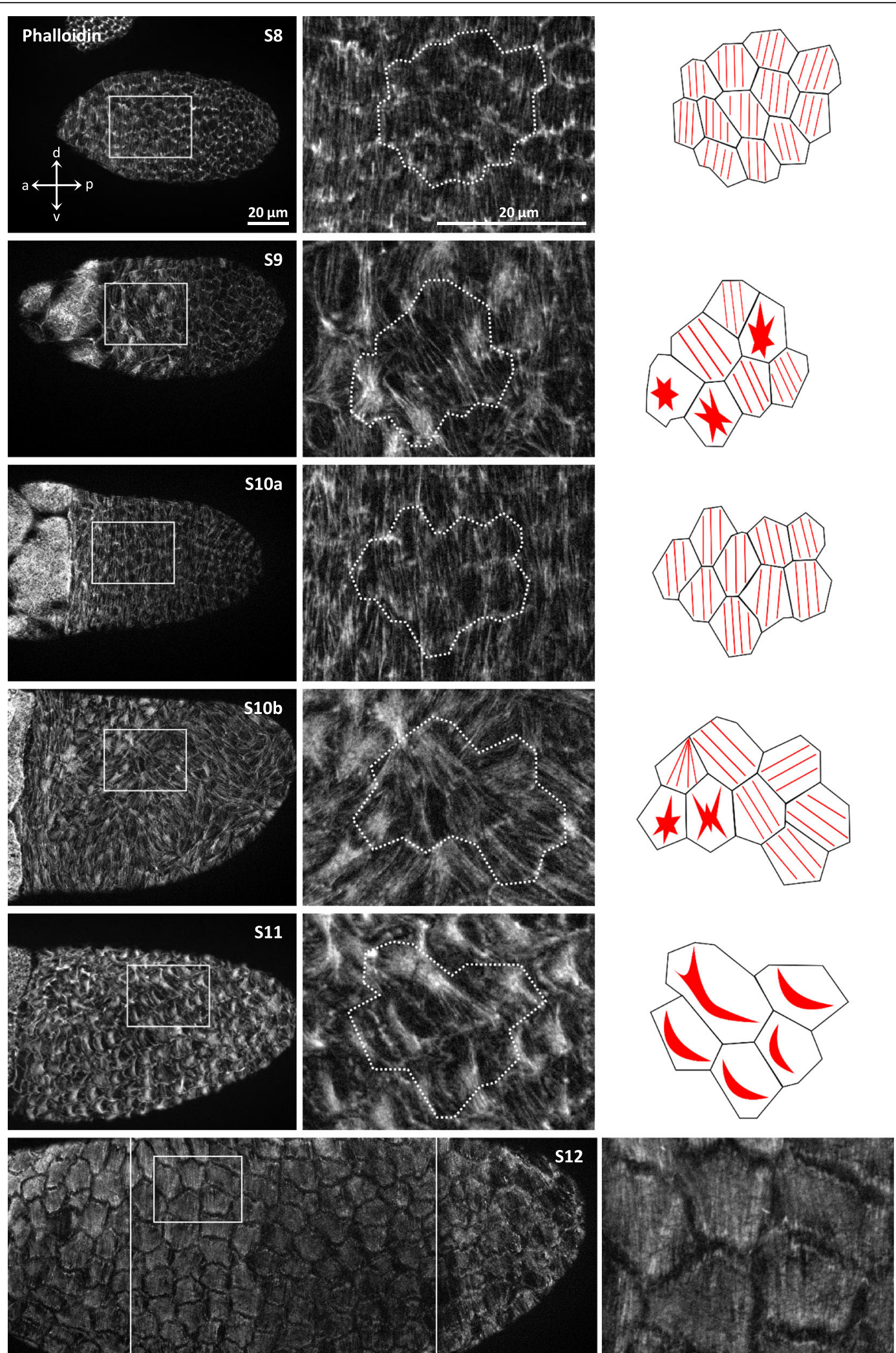

Fig. 4 (See legend on next page.) 
(See figure on previous page.)

Fig. 4 Development of the organisation of basal microfilaments (bMF) in the FCE during S8-12. Tangential optical sections (see Fig. 3) of typical follicles stained with fluorescent phalloidin are shown. Stage-specific features of the bMF-pattern are represented as sketches in the right column The dotted lines in the middle column (magnifications of boxed areas in the left column) correspond to the lateral FC-membranes seen at a deeper focal plane. Due to cell protrusions close to the basement membrane, the cell borders appear to be shifted. In the cuboidal FC of S8, the preferential bMF-bundle orientation within individual FC as well as relative to neighbouring FC is perpendicular to the follicle's antero-posterior (a-p) axis. During S9-11, rearrangements of the bMF-organisation occur. In S9, the bMF in the flattening CFC condense (red asterisks), whereas in S10a, the bMF-bundles in CFC are again aligned in parallel perpendicular to the a-p axis, i. e. along the dorso-ventral (d-v) axis. In S10b, condensation followed by disintegration of bMF become obvious in dorsal CFC as well as neighbouring FC, and this pattern spreads out toward the pFC in S11 (crescent-shaped condensations). During S12, a new pattern of dense parallel bMF perpendicular to the a-p axis emerges. In contrast to earlier stages, FC borders are discernible in this focal plane due to chorion ridges. For abbreviations, see legend to Fig. 1. Scale bars refer to all pictures in the same column

Bioelectrical patterns correlate with cytoskeletal patterns We have shown previously $[15,16]$ that, during the course of development, ovarian follicles undergo significant changes in their $\mathrm{pH}_{\mathrm{i}}$ - and $\mathrm{V}_{\text {mem- }}$-patterns caused by varying activities of asymmetrically distributed or activated ion-transport mechanisms. In the present study, we analysed in detail how the cytoskeletal organisation in the FCE alters during vitellogenesis (Figs. 4 and 5). It is obvious that stage-specific changes of $\mathrm{pH}_{\mathrm{i}}$ and $\mathrm{V}_{\text {mem }}$ correlate spatially and temporally with structural modifications of bMF and MT (summarised in Fig. 6). These alterations are accompanied by cell migrations, cell rearrangements, or cell-shape changes like, e.g., cell flattening or cell stretching. In S8, the uniformly cuboidal FCE exhibits relatively homogeneous $\mathrm{pH}_{\mathrm{i}}$ - and $\mathrm{V}_{\text {mem }}$-patterns as well as homogeneous bMF- and MT-patterns [16]. During $\mathrm{S} 9$, gradients of $\mathrm{pH}_{\mathrm{i}}$ and $\mathrm{V}_{\text {mem }}$ develop with relatively acidic and relatively depolarised cFC [16]. At this stage, the bMF in the flattening cFC lose their circumferential orientation and condense, while the MT change their orientation from $\mathrm{d}-\mathrm{v}$ to a-p. In S10a, the bMFbundles of the columnar FCE are aligned in parallel circumferentially again. During further development, $d-v$ gradients of $\mathrm{pH}_{\mathrm{i}}$ and $\mathrm{V}_{\text {mem }}$ develop [16]. In $\mathrm{S} 10 \mathrm{~b}$, the dorsal FCE is relatively hyperpolarised and relatively acidic compared to the ventral FCE and, as a result, dorsal cFC and neighbouring FC are the most acidic FC. In these, in part, inwardly migrating cells, the bMF condense again. During this process, in late S10b/11, a strong depolarisation of dorsal $\mathrm{cFC}$ and neighbouring FC becomes apparent. Unlike the bMF-pattern, the MTorganisation alters gradually along the a-p axis, but not along the $\mathrm{d}-\mathrm{v}$ axis. In $\mathrm{pFC}$, which are relatively alkaline and depolarised [16], no longitudinal alignment of MT was found.

\section{Modifying $\mathrm{pH}_{\mathrm{i}}$ and $\mathrm{V}_{\text {mem }}$ with inhibitors of ion-transport mechanisms}

We used six inhibitors of ion-transport mechanisms, which we have recently shown to affect either $\mathrm{pH}_{\mathrm{i}}, \mathrm{V}_{\text {mem }}$ or both parameters in the FCE during S10b [16]. We found that each tested inhibitor also exerted influence on the cytoskeletal organisation (Figs. 7, 8, 9 and 10). Certain groups of inhibitors giving rise to similar effects on $\mathrm{pH}_{\mathrm{i}}$ and/or $\mathrm{V}_{\text {mem }}$ caused similar changes in the bMFand/or MT-patterns. Therefore, we conclude that the observed cytoskeletal changes depended on the induced $\mathrm{pH}_{\mathrm{i}}$ - and/or $\mathrm{V}_{\text {mem }}$-changes, and not on effects of the involved ions.

As described in detail previously ([16], summarised in Fig. 2), alkalisation was caused by furosemide, glibenclamide, 9-anthroic acid or verapamil. Furosemide and glibenclamide resulted in the strongest overall increase of $\mathrm{pH}_{\mathrm{i}}$ and also in enhanced angles of the a-p and the $\mathrm{d}-\mathrm{v}$ gradient. 9-Anthroic acid led to an enhanced angle of the a-p gradient, but to a reduced angle of the $d-v$ gradient. Amiloride or bafilomycin resulted in acidification and in reduction of the angles of the $a-p$ and the $\mathrm{d}-\mathrm{v}$ gradient. $\mathrm{V}_{\text {mem }}$ was influenced to the greatest extent by verapamil (strong hyperpolarisation), followed by glibenclamide (hyperpolarisation), and both inhibitors reduced the angles of the a-p and the d-v gradient. Furosemide, 9-anthroic acid, amiloride and bafilomycin, respectively, had no consistent effects on $V_{\text {mem }}$ and on both gradients.

\section{Changes in $\mathrm{pH}_{\mathrm{i}}$ and $\mathrm{V}_{\text {mem }}$ affect the organisation of basal microfilaments}

Inhibition experiments were performed using S10bfollicles of the wild-type as well as of the transgenic strain Lifeact-GFP. The bMF-patterns in the FCE of both strains were very similar (Figs. 7 and 8 ), only a slight difference in the thickness of bMF-bundles was obvious: The bMF-bundles of fixed phalloidin-stained wild-type follicles were thinner than those of living Lifeact-GFP follicles. Furthermore, Lifeact-GFP follicles often showed a weakly fluorescent area in the FCE that seemed to result from squeezing during microscopic observation.

Despite of some variation, the effects of inhibitors on the bMF-patterns were also similar in both strains (Figs. 7 and 8). Strong alkalisation, either without a distinct effect on $V_{\text {mem }}$ (furosemide) or combined with hyperpolarisation (glibenclamide), retained a highly polarised 


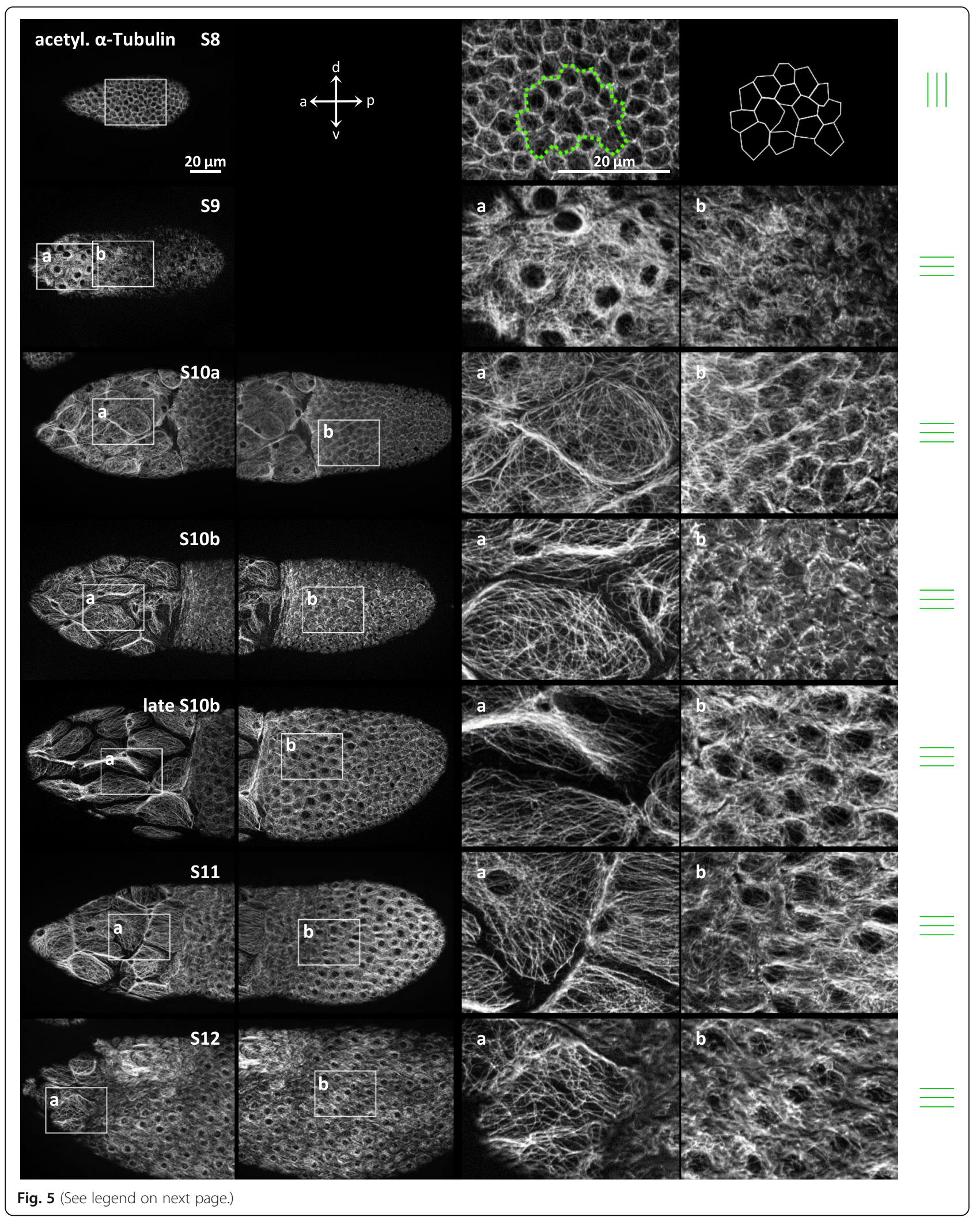


(See figure on previous page.)

Fig. 5 Development of the organisation of microtubules (MT) in the FCE during S8-12. Tangential optical sections (see Fig. 3) of typical antiacetylated a-tubulin-treated follicles are shown. In the cuboidal FC of S8, the preferential MT-orientation is, similar to the bMF-orientation, perpendicular to the follicle's a-p axis (indicated as parallel green lines at the right margin). To illustrate the location, shape and size of FC in S8, an area of the FCE (surrounded by a dotted green line) and the FC borders are highlighted in the right column. From S9 onward, the circumferential MT-orientation no longer exists. Diffuse MT enclose the FC nuclei (dark regions in the centre of the cells) in a basket-like arrangement. a During S9-12, the squamous FC covering the NC show a uniform organisation of MT. No preferential MT-orientation referred to the follicle's axes is discernible in these FC. $\mathbf{b}$ In the columnar FC, in addition to the basket-like arrangement, a longitudinal MT-orientation develops during S9-12 (parallel green lines at the right margin): In S9, the MT of CFC and mbFC first become oriented along the a-p axis of the follicle. From S10a-12, this pattern spreads out to the pFC. The two right columns show magnifications of boxed areas in the two left columns. For abbreviations, see legend to Fig. 1. Scale bars refer to all pictures in the same column

bMF-pattern consisting of parallel aligned, but thinner bMF-bundles, whereas condensations of bMF, as in the controls, were rarely observed (Fig. 8a,b). The bMFbundles of furosemide-treated follicles appeared to be even thinner and partially disintegrated compared to those of glibenclamide-treated follicles. Presumably, this difference depends on the fact that furosemide showed no clear influence on $V_{\text {mem }}$. Furosemide and glibenclamide both led to alkalisation in all FC, but especially in pFC and ventral FC, thus enhancing the angles of the a$\mathrm{p}$ and the $\mathrm{d}-\mathrm{v} \mathrm{pH}_{\mathrm{i}}$-gradient (cf. Figure 2). Both $\mathrm{V}_{\text {mem}^{-}}$ gradients were either maintained or reduced resulting in a larger area of relatively hyperpolarised FC.

Slight alkalisation together with no clear effect on $\mathrm{V}_{\text {mem }}$ (9-anthroic acid) reduced the frequency of bMFcondensations (Fig. 8a,b). This treatment led to an enhanced angle of the a-p $\mathrm{pH}_{\mathrm{i}}$-gradient but to a reduced angle of the $\mathrm{d}-\mathrm{v} \mathrm{pH}_{\mathrm{i}}$-gradient, since the ventral $\mathrm{cFC}$ became less alkalised (cf. Figure 2). Slight alkalisation combined with strong hyperpolarisation (verapamil) resulted either in depolymerisation or in condensation of bMF throughout the entire columnar FCE (Fig. 8b). This seems to be due to the fact that the angles of both the a$\mathrm{p}$ and the $\mathrm{d}-\mathrm{v} \mathrm{V}_{\mathrm{mem}}$-gradient were reduced, which led to more homogeneous electrochemical properties throughout the FCE (cf. Figure 2).

Acidification combined with an unchanged $\mathrm{V}_{\text {mem }}$ (amiloride, bafilomycin) led to an increase in bMF condensation and disintegration. The angles of the a-p and the $\mathrm{d}-\mathrm{v} \mathrm{pH}_{\mathrm{i}}$-gradient of both amiloride- and bafilomycintreated follicles were reduced (cf. Figure 2), and the relatively acidic area of the FCE showing condensed bMF was enlarged (Fig. 8a,b).

Taken together, we found that alkalisation prevented condensation of bMF and stabilised their parallel alignment, while the bMF-bundles became thinner. In contrast, acidification led to increasing condensations of bMF in both the a-p and the d-v direction, while the bMF-bundles became thicker and more disordered. When strong alkalisation was combined with hyperpolarisation, disintegration of bMF was absent. Thus, hyperpolarisation had a stabilising effect on bMF (Figs. 7 and 8).

\section{Changes in $\mathrm{pH}_{\mathrm{i}}$ and $\mathrm{V}_{\text {mem }}$ affect the organisation of microtubules}

Inhibition experiments were performed using S10bfollciles of the wild-type as well as of the transgenic strain $\alpha$ Tub84B-GFP. While, in living $\alpha$ Tub84B-GFP follicles, the $\alpha$-subunits of all MT in the FCE were labelled, only a subset of MT was stained in fixed wild-type follicles treated with an antibody against acetylated $\alpha$ tubulin. Thus, in $\alpha$ Tub84B-GFP, a denser network of MT-bundles was revealed and the overall longitudinal alignment of MT became more evident (Figs. 9 and 10).

Alkalisation, caused by furosemide, glibenclamide or 9-anthroic acid, resulted either in reduction (glibenclamide, 9-anthoric acid) or in loss (furosemide) of the longitudinal orientation of MT as well as in their partial disintegration. In furosemide-treated follicles (strong alkalisation, no clear effect on $V_{\text {mem }}$ ), disintegration of MT was most prominent compared to follicles treated with glibenclamide (strong alkalisation, slight hyperpolarisation) or with 9-anthoric acid (slight alkalisation, no clear effect on $\left.\mathrm{V}_{\mathrm{mem}}\right)$. Strong alkalisation combined with no clear effect on $V_{\text {mem }}$ (furosemide) resulted in spherical FC, presumably due to weakend cell-cell contacts, which was particularly visible in $\alpha$ Tub84B-GFP. This phenomenon was less pronounced with glibenclamide, presumably due to a stabilising effect of hyperpolarisation. Slight alkalisation combined with strong hyperpolarisation (verapamil) preserved the longitudinal orientation, while the MT-bundles appeared to be thicker (Figs. 9 and 10).

In addition, furosemide, glibenclamide or 9-anthroic acid led to an enhanced angle of the a- $\mathrm{pH}_{\mathrm{i}}$-gradient and to a reduced angle of the a-p $\mathrm{V}_{\text {mem }}$-gradient (cf. Figure 2). This means that the cFC became more alkaline compared to the $\mathrm{mbFC}$, while the area of relatively hyperpolarised FC became enlarged. The altered $\mathrm{pH}_{\mathrm{i}^{-}}$ gradient resulted in loss of the longitudinal MTalignment in the mbFC and cFC. Verapamil also led to an enhanced angle of the a- $\mathrm{pH}_{\mathrm{i}}$-gradient and to a reduced angle of the a-p $\mathrm{V}_{\text {mem-gradient (cf. Figure 2). But }}$ the effect of verapamil on $\mathrm{pH}_{\mathrm{i}}$ in general as well as on its gradients was small, so that it had no impact on MTorganisation. In addition, the strong hyperpolarising 


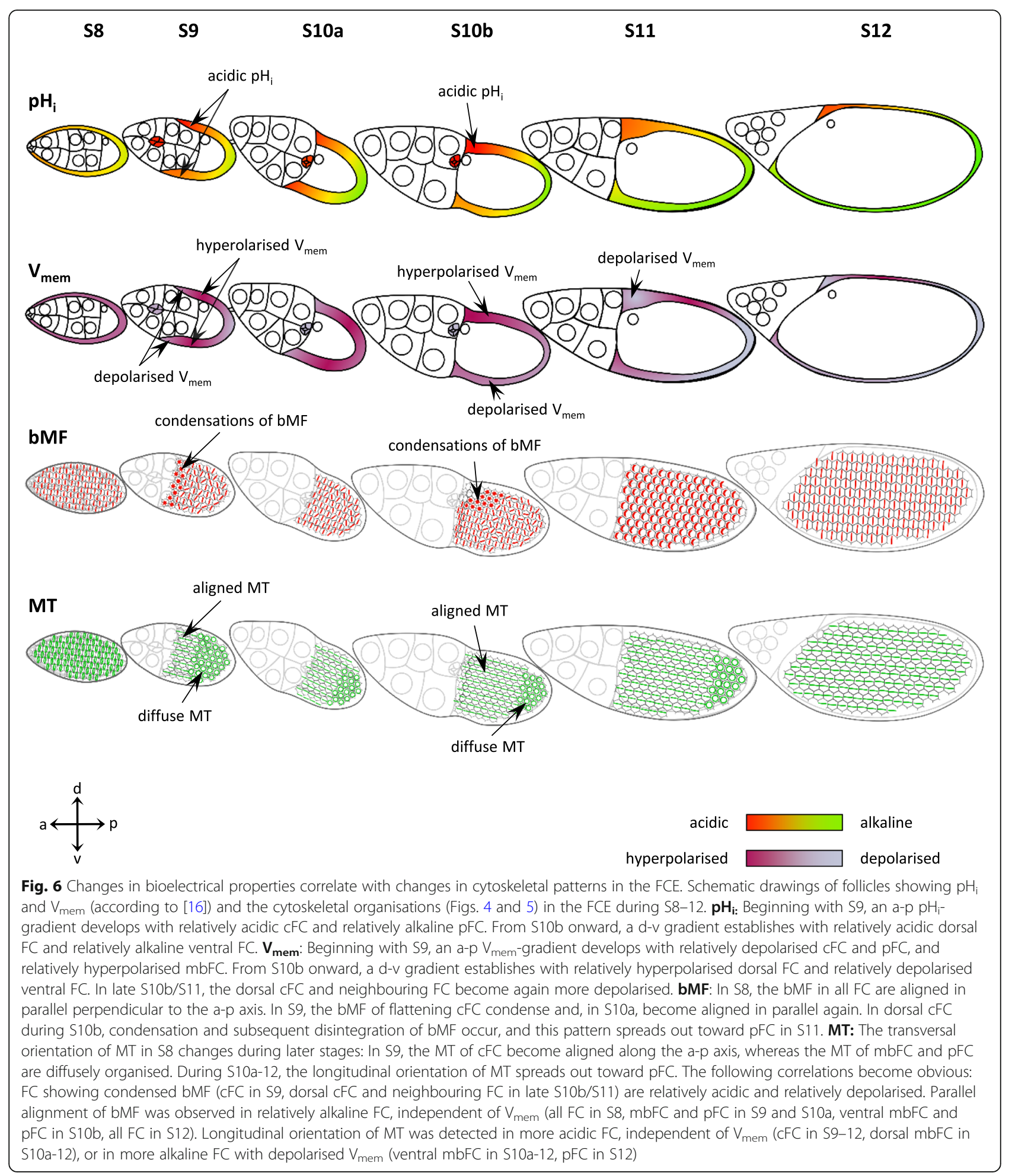

effect of verapamil and the reduced $\mathrm{V}_{\text {mem }}$-gradients both preserved the longitudinal alignment of MT (Figs. 9 and 10).

Acidification in the whole FCE as well as reduced angles of both $\mathrm{pH}_{\mathrm{i}}$-gradients combined with no clear effects on $\mathrm{V}_{\text {mem }}$ caused by amiloride and bafilomycin (cf. Figure 2) did not alter the MT-organisation (not shown).

Therefore, we conclude that hyperpolarisation as well as acidification exerted stabilising effects on the longitudinal orientation of MT-bundles. Strong alkalisation 


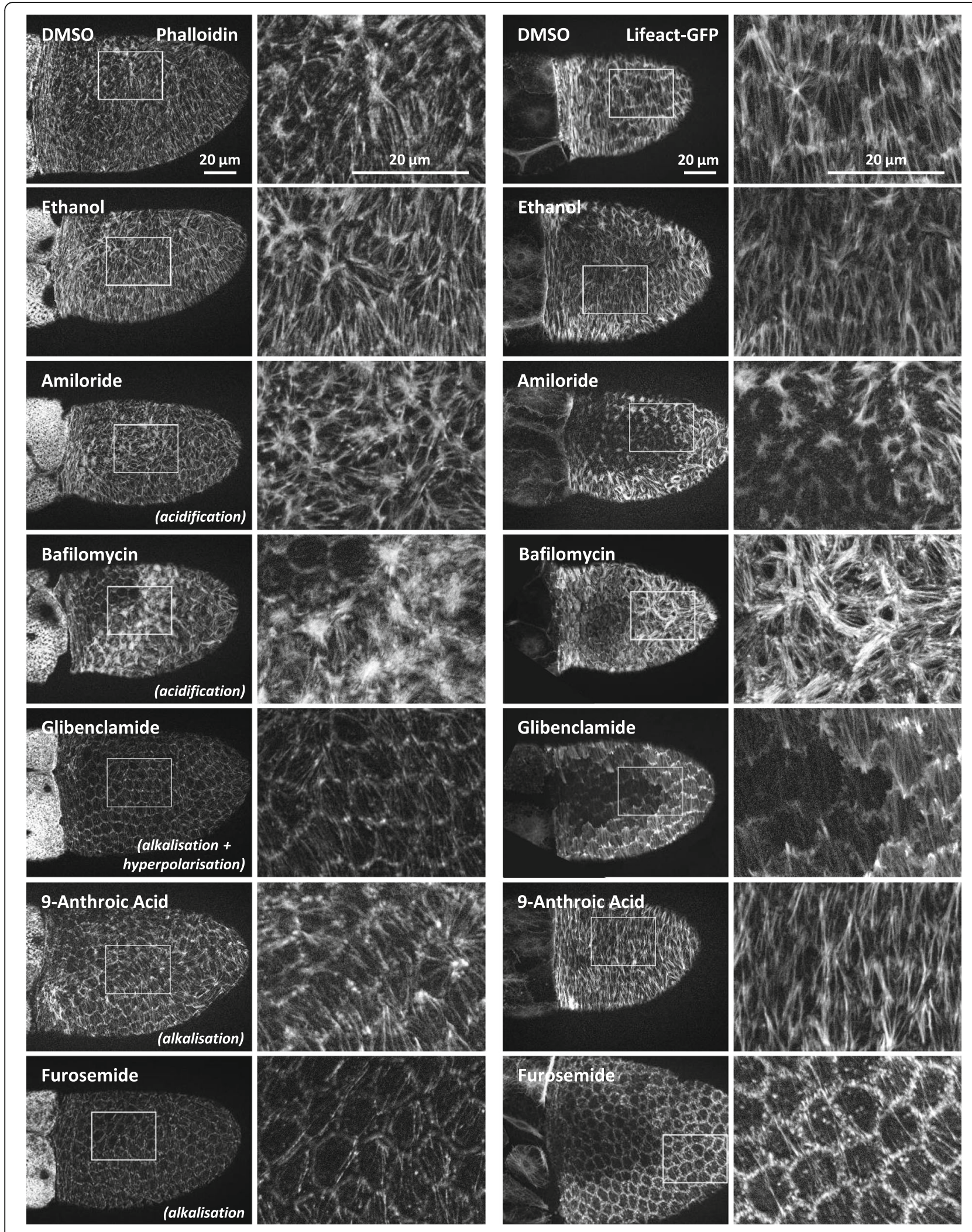

Fig. 7 (See legend on next page.) 
(See figure on previous page.)

Fig. 7 The bMF-organisation is affected by alterations of both $\mathrm{pH}_{\mathrm{i}}$ and $\mathrm{V}_{\text {mem. }}$. Using inhibitors of ion-transport mechanisms, we modified $\mathrm{pH}$ or $V_{\text {mem }}$ as well as the bMF-pattern. The results obtained with living Lifeact-GFP follicles (right columns) were similar to those obtained with fixed wild-type follicles using fluorescent phalloidin (left columns). Typical follicles of S10b are shown. For summary, see Fig. 8. Scale bars refer to all pictures in the same column. The inhibitors glibenclamide (ATP-sensitive $\mathrm{K}^{+}$-channels) or furosemide $\left(\mathrm{Na}^{+} / \mathrm{K}^{+} / 2 \mathrm{Cl}^{-}\right.$-cotransporters), which both caused strong alkalisation (cf. Figure 2), resulted in parallel alignment of bMF in all FC (control DMSO). Glibenclamide, which led to (moderate) hyperpolarisation, stabilised the bMF-bundles, while furosemide, which had no clear effect on $\mathrm{V}_{\text {mem }}$, caused partial disintegration of bMF. The inhibitor 9-anthroic acid ( $\mathrm{Cl}^{-}$-channels), which resulted in slight alkalisation and no clear effect on $\mathrm{V}_{\text {mem }}$ (cf. Figure 2), also reduced the frequency of bMF-condensations (control ethanol). On the other hand, bafilomycin (V-ATPases) or amiloride $\left(\mathrm{Na}^{+} / \mathrm{H}^{+}\right.$-exchangers, $\mathrm{Na}^{+}$-channels), both acidifying inhibitors with no strong impact on $V_{\text {mem }}$ (cf. Figure 2), led to an increasing area of bMF-condensation followed by disintegration of bMF in CFC and dorsal mbFC

resulted in loss of this MT-arrangement and in partial disintegration of MT. These effects were reduced when alkalisation was combined with hyperpolarisation, which preserved the longitudinal orientation of MT-bundles (Figs. 9 and 10).

\section{Discussion}

Considering the results of our analysis of stage-specific patterns as well as of inhibition experiments, correlations between $\mathrm{pH}_{\mathrm{i}}$ - and $\mathrm{V}_{\text {mem }}$-changes and changes of the cytoskeletal organisation become obvious. Alkalisation supports the parallel alignment of bMF-bundles and prevents the longitudinal orientation of MT, whereas acidification results in increasing condensation and subsequent disintegration of bMF while supporting the longitudinal alignment of MT. Depending on $\mathrm{pH}_{\mathrm{i}}$, hyperpolarisation has stabilising effects on bMF- or on MT-bundles, whereas depolarisation correlates with bMF-disintegration or with reduced longitudinal MTorientation (summarised in Fig. 11). Obviously, bMF and MT are not disintegrated under the same electrochemical conditions. It seems as if bMF are predominantly stabilising the transversal axis while MT are stabilising the longitudinal axis of the follicle.

Our observations are in line with previous findings in various systems. Besides a $\mathrm{pH}$-dependence of actin selfassembly [23], it has been shown that contractility of the actomyosin cytoskeleton [24] as well as the activities of cross-linking proteins, like cortexillin, filamin and fascin, are regulated by $\mathrm{pH}[25,26]$. The interplay between actin, myosin and cross-linking proteins is highly complex, and the $\mathrm{pH}$-dependencies of these proteins are diverse. Accordingly, specific changes of the cytoskeletal organisation in consequence of $\mathrm{pH}_{\mathrm{i}}$-modifications relate to the respective cytoplasmic mixture of actin-binding proteins [25]. Also indirect effects, e.g. changes in cell volume or cell tension, could be envolved.

Condensation of bMF was mainly observed in FC that undergo significant morphological changes, like flattening and stretching. This applies for the cFC in S9 and for the dorsal $\mathrm{CFC}$ and neighbouring $\mathrm{FC}$, the prospective floor and roof cells of respiratory appendages [54, 55], in S10b. Since cell-shape changes require a reorganisation of the cytoskeleton, we assume that condensation and subsequent disintegration of bMF are distinct steps of cytoskeletal restructuring processes. Reorganisation of the bMF-network is usually correlated with lower $\mathrm{pH}_{\mathrm{i}}$. It has been shown in vitro that acidification leads to increased bundling of MF and to subsequent contraction of the actomyosin network, while alkalisation maintains the MF-organisation by stabilising cross-linking [24]. The described condensation of bMF in the relatively acidic $\mathrm{cFC}$ in $\mathrm{S} 9$ and dorsal cFC in S10b, as well as in the mbFC after treatment with amiloride or bafilomycin, seems to be based on a comparable mechanism. On the other hand, the parallel alignment of bMF in relatively alkaline FC, for example after application of glibenclamide or furosemide, indicates stable cross-linking between bMFbundles. This interpretation is further supported by the observation that actin self-assembly is accelerated at lower $\mathrm{pH}_{\mathrm{i}}$ [23], since restructuring of the MFnetwork depends on rapid self-assembly.

The MT-organisation has been shown to be directly influenced by $\mathrm{pH}_{\mathrm{i}}$-changes: Acidification results in polymerisation of tubulin while alkalisation results in depolymerisation $[25,29,30]$. Loss of the longitudinal alignment of $\mathrm{MT}$ observed in the relatively alkaline pFC and in all FC after the application of alkalising inhibitors (furosemide, glibenclamide or 9-anthroic acid) is likely to be based on MT-depolymerisation (Fig. 11).

In addition to being regulated by $\mathrm{pH}_{\mathrm{i}}$-changes, both $\mathrm{MF}$ and $\mathrm{MT}$ are known to be affected by $\mathrm{V}_{\text {mem }^{-}}$ changes. In cultured bovine corneal endothelial cells, it has been demonstrated that depolarisation of $V_{\text {mem }}$ leads to reorganisation and to decreasing densities of the MF- and MT-networks [31]. Depolarisation is usually correlated with MF-network restructuring processes, whereas hyperpolarisation is correlated with stabilisation of the MF-organisation [27, 28]. These findings are in line with our own observations (Fig. 11). Moreover, since MF as well as MT are charged and polar polymers that can act as electrical conductors, both cytoskeletal networks are highly sensitive to electrical fields [32, 33, 56]. 

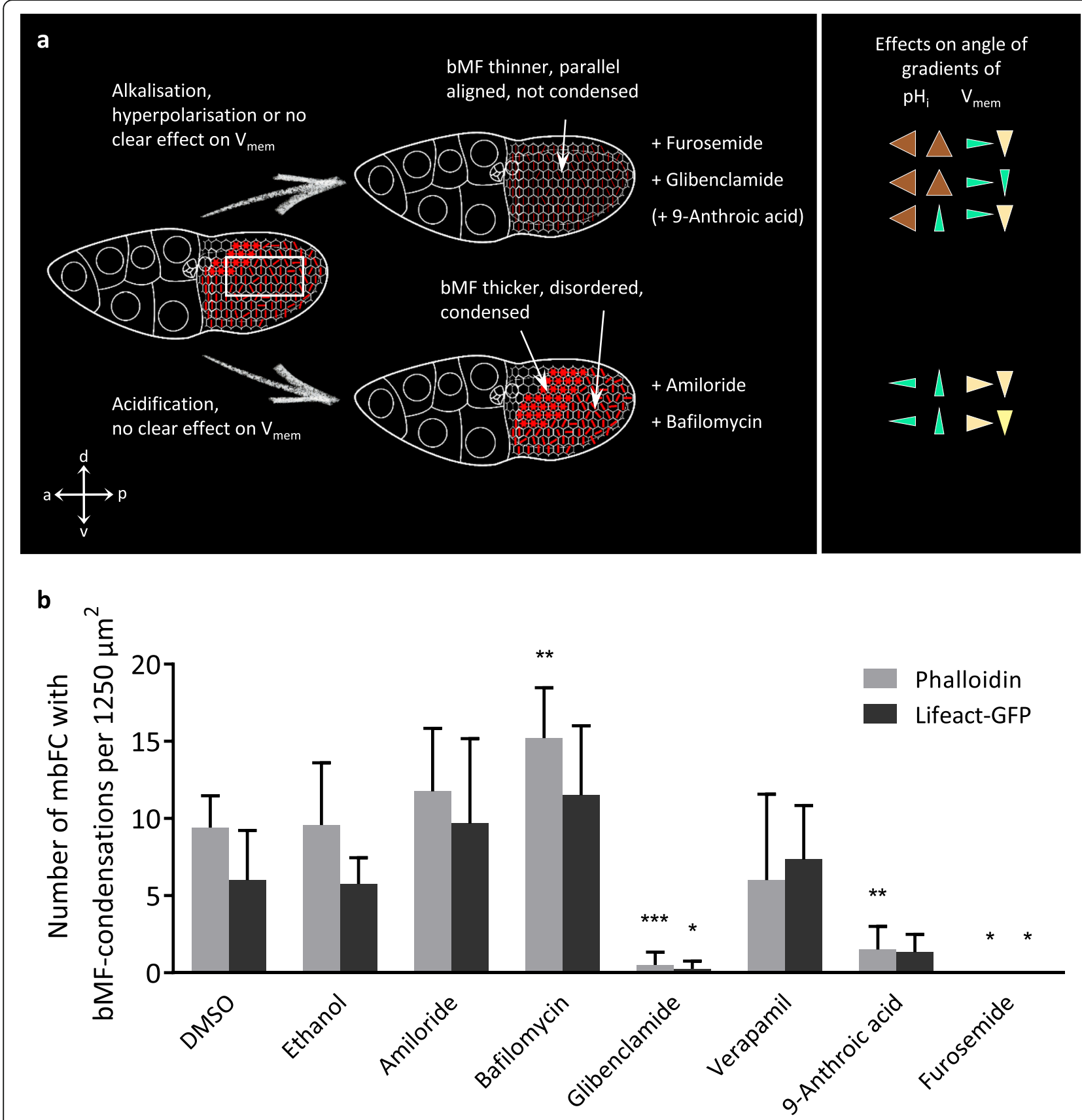

Fig. 8 Schematic summary of effects of $\mathrm{pH}_{i}$ and/or $\mathrm{V}_{\text {mem }}$ on bMF, and quantification of bMF-condensations (cf. Figure 7). a Alkalisation (together with hyperpolarisation or no clear effect on $V_{\text {mem }}$ ) caused by glibenclamide, furosemide or 9-anthroic acid prevented condensation of bMF (see b) and stabilised their parallel alignment, while the bMF-bundles became thinner. This was accompanied by enhanced angles of $\mathrm{pH}_{\mathrm{i}}$-gradients and reduced angles of $V_{\text {mem }}$-gradients (shown on the right, $\mathrm{cf}$. Figure 2). In contrast, acidification (together with no clear effect on $V_{\text {mem }}$ ), caused by amiloride or bafilomycin, led to an increasing area of bMF-condensation (see $\mathbf{b}$ ) in both the a-p and the $d-v$ direction. Moreover, bMF-bundles appeared to be thicker and more disordered. This was accompanied by reduced angles of $\mathrm{pH}_{\mathrm{i}}$-gradients and no clear effects on angles of $\mathrm{V}_{\text {mem }}{ }^{-}$ gradients (shown on the right, cf. Figure 2). b Quantification of bMF-condensations in mbFC (for region of interest, see box marked in a) supported the results described above. Mean values, shown with their standard deviation, were compared with the respective controls using an unpaired t-test $\left(3 \leq n \leq 8 ;{ }^{*} p<0.05 ;{ }^{* *} p<0.01 ;{ }^{* * *} p<0.001\right)$. Verapamil (slight alkalisation combined with strong hyperpolarisation) resulted either in condensation or in depolymerisation of bMF 

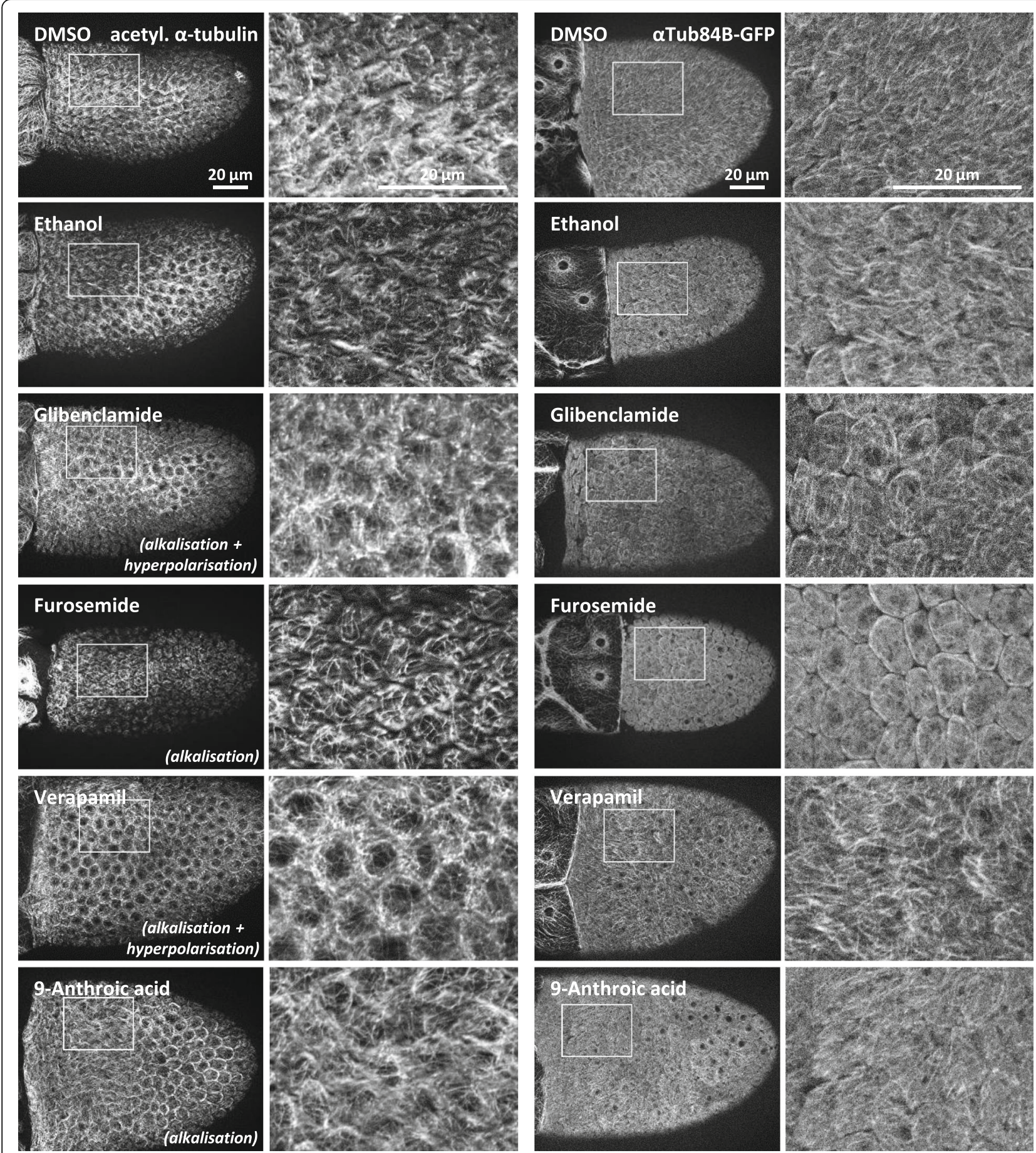

Fig. 9 The MT-organisation is affected by alterations of both $\mathrm{pH}_{\mathrm{i}}$ and $\mathrm{V}_{\text {mem. }}$. Using inhibitors of ion-transport mechanisms, we modified $\mathrm{pH} \mathrm{H}_{\mathrm{i}}$ and/ or $V_{\text {mem }}$ as well as the MT-pattern. The results obtained with living aTub84B-GFP follicles (right columns) were similar to those obtained with fixed wild-type follicles using an antibody against acetylated a-tubulin (left columns). Typical follicles of S10b are shown. For summary, see Fig. 10. Scale bars refer to all pictures in the same column. The inhibitor verapamil (voltage-dependent L-type $\mathrm{Ca}^{2+}$-channels), which had the strongest impact on $V_{\text {mem }}$ (strong hyperpolarisation, reduction of the angles of the a-p and the $d-v$ gradient, cf. Figure 2) and led to alkalisation, stabilised the longitudinal alignment of MT. In addition, the MT-bundles appeared to be thicker than in the control (ethanol). On the other hand, the MT of follicles treated with either furosemide $\left(\mathrm{Na}^{+} / \mathrm{K}^{+} / 2 \mathrm{Cl}^{-}\right.$-cotransporters; no clear effect on $\mathrm{V}_{\text {mem; }}$ strong alkalisation, cf. Figure 2) or glibenclamide (ATP-sensitive $\mathrm{K}^{+}$-channels; moderate hyperpolarisation; strong alkalisation, cf. Figure 2) lost their longitudinal alignment and became partially disintegrated. This coincided with a spherical FC shape, which was especially noticeable in aTub84B-GFP (control DMSO). With 9-anthroic acid $\left(\mathrm{Cl}^{-}\right.$-channels; no clear effect on $\mathrm{V}_{\text {mem; }}$ slight alkalisation, cf. Figure 2), this effect was weaker (control ethanol) 


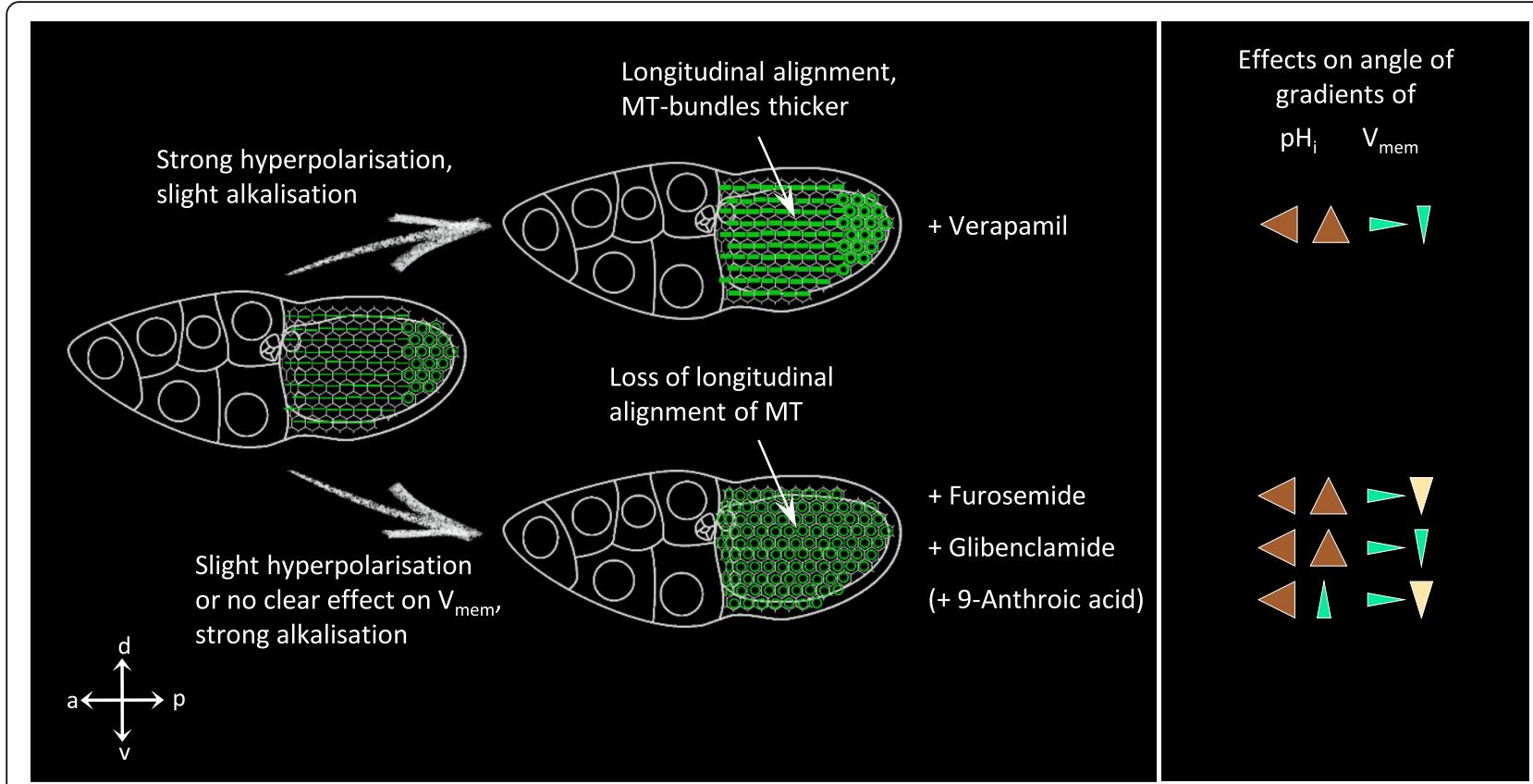

Fig. 10 Schematic summary of effects of $\mathrm{pH}_{i}$ and/or $\mathrm{V}_{\text {mem }}$ on MT (cf. Figure 9). Alkalisation (together with slight hyperpolarisation or no clear effect on $V_{\text {mem }}$ ) caused by furosemide, glibenclamide or 9-anthroic acid led to diffuse MT in all FC and to loss of their longitudinal alignment. This was accompanied by enhanced angles of $\mathrm{pH}_{\mathrm{i}}$-gradients and reduced angles of $\mathrm{V}_{\text {mem-gradients }}$ (shown on the right, cf. Figure 2). Slight alkalisation together with strong hyperpolarisation as well as enhanced angles of $\mathrm{pH}_{\mathrm{i}}$-gradients and reduced angles of $\mathrm{V}_{\text {mem-gradients }}$ (verapamil; shown on the right, cf. Figure 2) maintained the longitudinal MT-organisation. This was complemented by thickening of the MT-bundles

\section{Conclusion}

Correlations between stage-specific bioelectrical properties and cytoskeletal patterns observed in the FCE of Drosophila were confirmed by the application of inhibitors of several ion-transport mechanisms. We conclude that the changes of $\mathrm{pH}_{\mathrm{i}^{-}}$and $\mathrm{V}_{\text {mem }}$-gradients induced by inhibitors simulate electrochemical changes that occur naturally, resulting in the cytoskeletal changes observed during differentiation of the FCE. Our results support the hypothesis that electrochemical signals play important roles in the regulation of cell and tissue architecture by organising elements of the MF- and MTcytoskeleton. It remains to be shown which specific elements are affected by these signals.

\section{Methods}

\section{Preparation of follicles}

Drosophila melanogaster were reared at $20-23^{\circ} \mathrm{C}$ on standard medium with additional fresh yeast. $2-3$ days old females were killed by crushing the head and thorax with tweezers without anaesthesia. The ovaries were dissected and single follicles of vitellogenic stages (S8-12) were isolated (see Fig. 1). The preparations were carried out in R14 medium [57] which is best suited for in-vitro culture of Drosophila follicles [58].

In addition to wild-type (Oregon R), we used the Gal4/UAS system for the follicle-cell specific expression
(Tj-Gal4; gift of S. Roth, Köln, Germany) of GFP-actin (UAS-Lifeact-GFP; Bloomington Stock Center, USA) and GFP- $\alpha$-tubulin (UAS- $\alpha$ Tub84B; Bloomington), respectively.

\section{Labelling of microfilaments}

Follicles were fixed for $20 \mathrm{~min}$ in microfilamentstabilising buffer (MF-buffer) containing 4\% formaldehyde according to [47], washed in phosphate-buffered saline (PBS) and stained for $20 \mathrm{~min}$ with $0.25 \mu \mathrm{g} / \mathrm{ml}$ phalloidin-FluoProbes 550A (Interchim, France; dissolved in dimethyl sulfoxide, DMSO) which specifically binds to F-actin.

\section{Indirect immunofluorescence labelling of microtubules}

Follicles were fixed for $20 \mathrm{~min}$ in MF-buffer, washed in PBS and blocked for $1 \mathrm{~h}$ with $2 \%$ bovine serum albumin (BSA)/0.1\% Triton X-100 in PBS. Thereafter, the follicles were incubated for $1 \mathrm{~h}$ at $20^{\circ} \mathrm{C}$ or overnight at $4{ }^{\circ} \mathrm{C}$ in PBS containing $1 \%$ BSA $/ 0.1 \%$ Triton X-100 and a monoclonal antibody against acetylated $\alpha$-tubulin $(6-11 \mathrm{~B}-1$; Santa Cruz Biotechnology, USA; diluted 1:100). After washing, the follicles were treated with goat-antimouse biotin (Dianova, Germany; diluted 1:200) for 1 $\mathrm{h}$ in PBS containing $1 \%$ BSA/0.1\% Triton X-100. Washing was repeated before TexasRed-conjugated 


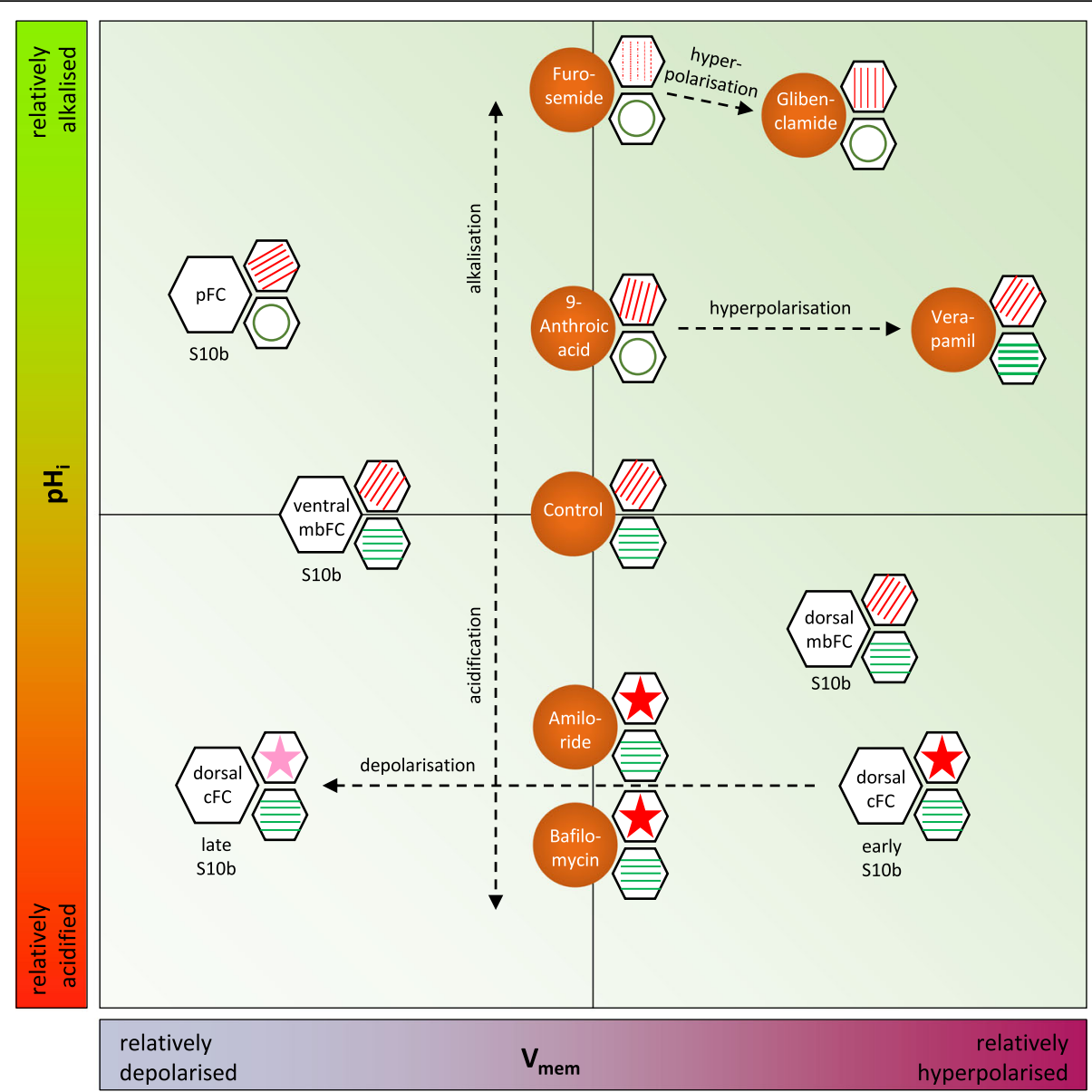

Fig. 11 Schematic representation of correlations between $\mathrm{pH}_{\mathrm{i}}, \mathrm{V}_{\mathrm{mem}}, \mathrm{bMF}$ and MT. The different FC types in S10b (cf. Figure 1) are symbolised by white hexagons, and the applied inhibitors by orange circles. The organisation of bMF in FC is symbolised by red lines (parallel orientation: $d-v$ or oblique; dotted: disintegration) or by red asterisks in hexagons (dark red: condensation; light red: condensation and disintegration). The organisation of MT in FC is symbolised by green lines (parallel orientation: a-p; thick line: thickening) or by green circles in hexagons (loss of parallel orientation and partial disintegration). Dotted arrows indicate changes of $\mathrm{pH}_{\mathrm{i}}$ (relative acidification or alkalisation; left margin) and changes of $\mathrm{V}_{\text {mem }}$ (relative hyperpolarisation or depolarisation; bottom margin) relative to the control state (centre). The correlations between $\mathrm{pH}_{\mathrm{i}}$, $V_{\text {mem, }}$, bMF and MT observed in different FC types during S10b are in line with the correlations resulting from the inhibition of $\mathrm{Na}^{+} / \mathrm{H}^{+}$exchangers and $\mathrm{Na}^{+}$-channels (amiloride), V-ATPases (bafilomycin), ATP-sensitive $\mathrm{K}^{+}$-channels (glibenclamide), voltage-dependent $\mathrm{L}_{\text {-type }} \mathrm{Ca}^{2+}$ channels (verapamil), $\mathrm{Cl}^{-}$-channels (9-anthroic acid) or $\mathrm{Na}^{+} / \mathrm{K}^{+} / 2 \mathrm{Cl}^{-}$-cotransporters (furosemide). While alkalisation and/or hyperpolarisation stabilises the parallel transversal alignment of bMF, acidification leads to increasing disorder and to condensations of bMF. On the other hand, acidification as well as hyperpolarisation stabilises the longitudinal orientation of MT, whereas alkalisation leads to loss of this arrangement and to partial disintegration

streptavidin (Dianova; diluted 1:1000) was added for 30 min in PBS containing $1 \%$ BSA/0.1\% Triton X-100.

\section{Fluorescence microscopy and optical sectioning}

Fixed follicles were imaged in Fluoromount G (Interchim), and living follicles in R-14 medium, respectively, using a Zeiss AxioImager.M2 structured-illumination microscope, equipped with a Zeiss ApoTome and a Zeiss AxioCamMRm camera using a $\times 40 / 1.3$ oil objective and the appropriate filter sets. To investigate either basal microfilament (bMF) or microtubule (MT) patterns, tangential optical sections of follicles were analysed (Fig. 3).

\section{Inhibition of ion-transport mechanisms}

All S10b-follicles of a single fly (approximately 10-20 follicles) were divided into a control group and an experimental group. Inhibition was performed for $20 \mathrm{~min}$ in $\mathrm{R}-14$ medium containing the respective inhibitor according to [16]. The following inhibitors of ion-transport mechanisms were used: $\mathrm{Na}^{+} / \mathrm{H}^{+}$-exchangers (NHE) and $\mathrm{Na}^{+}$-channels were blocked with amiloride (Sigma-Aldrich, Germany; $10 \mu \mathrm{M}$; dissolved in DMSO), VATPases with bafilomycin A1 (Sigma-Aldrich; $160 \mathrm{nM}$; dissolved in DMSO), ATP-sensitive $\mathrm{K}^{+}$-channels with glibenclamide (Biomol, Germany; $100 \mu \mathrm{M}$; dissolved in DMSO), voltage-dependent $\mathrm{L}$-type $\mathrm{Ca}^{2+}$-channels with 
verapamil- $\mathrm{HCl}$ (Sigma-Aldrich; $50 \mu \mathrm{M}$; dissolved in ethanol), $\mathrm{Cl}^{-}$-channels with 9-anthroic acid (Sigma-Aldrich; $100 \mu \mathrm{M}$; dissolved in ethanol), and $\mathrm{Na}^{+} / \mathrm{K}^{+} / 2 \mathrm{Cl}^{-}$-cotransporters with furosemide (Sigma-Aldrich; $1 \mathrm{mM}$; dissolved in DMSO). Control experiments were performed in R-14 medium containing $0.1-1 \% \mathrm{v} / \mathrm{v}$ ethanol or DMSO without the respective inhibitor. After treatment, wild-type follicles were fixed and stained before analysis while GFP-follicles were directly analysed as described above. Each experiment was performed at least three times.

\begin{abstract}
Abbreviations
a-p: Antero-posterior; bMF: Basal microfilaments; cFC: Centripetal follicle cells; DIC: Differential interference contrast; DMSO: Dimethyl sulfoxide; $d-v$ : Dorsoventral; FC: Follicle cell; FCE: Follicle-cell epithelium; mbFC: Mainbody follicle cells; MF: Microfilaments; MF-buffer: Microfilament-stabilising buffer; MT: Microtubules; NC: Nurse cells; NHE: $\mathrm{Na}^{+} / \mathrm{H}^{+}$-exchangers; Oo: Oocyte; PBS: Phosphate buffered saline; pFC: Posterior follicle cells; $\mathrm{pH}_{\mathrm{i}}$ : Intracellular $\mathrm{pH}$; S: Stage; $\mathrm{V}_{\text {mem }}$ : Membrane potential
\end{abstract}

\section{Acknowledgements}

We thank Susanne Schotthöfer for technical assistance, and Siegfried Roth (Köln, Germany) for providing the Tj-Gal4 strain. UAS-Lifeact-GFP and UASaTub84B were obtained from the Bloomington Drosophila Stock Center (USA).

\section{Authors' contributions}

IW carried out the experiments and analysed the data under the supervision of JB. JB conceived the study and reviewed the data. Both authors wrote the manuscript and read and approved the final version.

\section{Funding}

Financial support by RWTH Aachen University is acknowledged. The funding body played no role in the design of the study or the collection, analysis, and interpretation of data, or in writing the manuscript.

\section{Availability of data and materials}

The datasets used during the current study are available from the corresponding author on reasonable request.

\section{Ethics approval and consent to participate}

Not applicable.

\section{Consent for publication}

Not applicable.

\section{Competing interests}

The authors declare that they have no competing interests.

Received: 13 July 2019 Accepted: 24 October 2019

\section{Published online: 12 November 2019}

\section{References}

1. McCaig CD, Rajnicek AM, Song B, Zhao M. Controlling cell behavior electrically: current views and future potential. Physiol Rev. 2005;85:943-78.

2. Chang F, Minc N. Electrochemical control of cell and tissue polarity. Annu Rev Cell Dev Biol. 2014;30:317-36.

3. Levin M, Pezzulo G, Finkelstein JM. Endogenous bioelectric signaling networks: exploiting voltage gradients for control of growth and form. Annu Rev Biomed Eng. 2017;19:353-87.

4. McLaughlin KA, Levin M. Bioelectric signaling in regeneration: mechanisms of ionic controls of growth and form. Dev Biol. 2018;433:177-89.

5. Pietak A, Levin M. Bioelectrical control of positional information in development and regeneration: a review of conceptual and computational advances. Prog Biophys Mol Biol. 2018;137:52-68.

6. Cervera J, Manzanares JA, Mafe S. Cell-cell bioelectrical interactions and local heterogeneities in genetic networks: a model for the stabilization of single-cell states and multicellular oscillations. Phys Chem Chem Phys. 2018; 20:9343-54.

7. Blackiston DJ, McLaughlin KA, Levin M. Bioelectric controls of cell proliferation: ion channels, membrane voltage and the cell cycle. Cell Cycle. 2009;8:3527-36

8. Stock C, Ludwig FT, Hanley PJ, Schwab A. Roles of ion transport in control of cell motility. Compr Physiol. 2013;3:59-119.

9. Ulmschneider B, Grillo-Hill BK, Benitez M, Azimova DR, Barber DL, Nystul TG. Increased intracellular $\mathrm{pH}$ is necessary for adult epithelial and embryonic stem cell differentiation. J Cell Biol. 2016;215:345-55.

10. Tatapudy S, Aloisio F, Barber D, Nystul T. Cell fate decisions: emerging roles for metabolic signals and cell morphology. EMBO Rep. 2017;18:2105-18.

11. George LF, Pradhan SJ, Mitchell D, Josey M, Casey J, Belus MT, Fedder KN, Dahal GR, Bates EA. Ion channel contributions to wing development in Drosophila melanogaster. G3 (Bethesda). 2019;9:999-1008.

12. Benitez M, Tatapudy S, Liu Y, Barber DL, Nystul TG. Drosophila anion exchanger 2 is required for proper ovary development and oogenesis. Dev Biol. 2019;452:127-33.

13. Adams DS, Levin M. Endogenous voltage gradients as mediators of cell-cell communication: strategies for investigating bioelectrical signals during pattern formation. Cell Tissue Res. 2013;352:95-122.

14. Levin M. Endogenous bioelectrical networks store non-genetic patterning information during development and regeneration. J Physiol. 2014;592: 2295-305.

15. Krüger J, Bohrmann J. Bioelectric patterning during oogenesis: stage-specific distribution of membrane potentials, intracellular $\mathrm{pH}$ and ion-transport mechanisms in Drosophila ovarian follicles. BMC Dev Biol. 2015;15:1.

16. Weiß I, Bohrmann J. Electrochemical patterns during Drosophila oogenesis: ion-transport mechanisms generate stage-specific gradients of $\mathrm{pH}$ and membrane potential in the follicle-cell epithelium. BMC Dev Biol. 2019;19:12.

17. Bohrmann J, Haas-Assenbaum A. Gap junctions in ovarian follicles of Drosophila melanogaster: inhibition and promotion of dye-coupling between oocyte and follicle cells. Cell Tissue Res. 1993;273:163-73.

18. Bohrmann J, Zimmermann J. Gap junctions in the ovary of Drosophila melanogaster: localization of innexins 1, 2,3 and 4 and evidence for intercellular communication via innexin-2 containing channels. BMC Dev Biol. 2008;8:111.

19. Lautemann J, Bohrmann J. Relating proton pumps with gap junctions: Colocalization of ductin, the channel-forming subunit c of V-ATPase, with subunit a and with innexins 2 and 3 during Drosophila oogenesis. BMC Dev Biol. 2016;16:24.

20. Mathews J, Levin M. Gap junctional signaling in pattern regulation: physiological network connectivity instructs growth and form. Dev Neurobiol. 2017;77:643-73.

21. Cervera J, Pietak A, Levin M, Mafe S. Bioelectrical coupling in multicellular domains regulated by gap junctions: a conceptual approach. Bioelectrochemistry. 2018;123:45-61.

22. Campetelli A, Bonazzi D, Minc N. Electrochemical regulation of cell polarity and the cytoskeleton. Cytoskeleton. 2012;69:601-12.

23. Wang F, Sampogna RV, Ware BR. pH dependence of actin self-assembly. Biophys J. 1989;55:293-8.

24. Köhler S, Schmoller KM, Crevenna AH, Bausch AR. Regulating contractility of the actomyosin cytoskeleton by pH. Cell Rep. 2012;2:433-9.

25. Busa WB. Mechanisms and consequences of $\mathrm{pH}$-mediated cell regulation. Annu Rev Physiol. 1986:48:389-402

26. Schmoller KM, Köhler S, Crevenna AH, Wedlich-Soldner R, Bausch AR. Modulation of cross-linked actin networks by pH. Soft Matter. 2012;8:9685-90.

27. Chifflet $\mathrm{S}$, Hernández JA. The plasma membrane potential and the organization of the actin cytoskeleton of epithelial cells. Int J Cell Biol. 2012; 2012:121424.

28. Callies C, Fels J, Liashkovich I, Kliche K, Jeggle P, Kusche-Vihrog K, Oberleithner $\mathrm{H}$. Membrane potential depolarization decreases the stiffness of vascular endothelial cells. J Cell Sci. 2011;124:1936-42.

29. Regula CS, Pfeiffer JR, Berlin RD. Microtubule assembly and disassembly at alkaline pH. J Cell Biol. 1981;89:45-53.

30. de Brabander M, Geuens G, Nuydens R, Willebrords R, de Mey J. Microtubule stability and assembly in living cells: the influence of metabolic inhibitors, taxol and pH. Cold Spring Harb Symp. 1982;46:227-40.

31. Chifflet S, Hernández JA, Grasso S, Cirillo A. Nonspecific depolarization of the plasma membrane potential induces cytoskeletal modifications of bovine corneal endothelial cells in culture. Exp Cell Res. 2003;282:1-13. 
32. Priel A, Ramos AJ, Tuszynski JA, Cantiello HF. A biopolymer transistor: electrical amplification by microtubules. Biophys J. 2006;90:4639-43.

33. Cantero MDR, Villa Etchegoyen C, Perez PL, Scarinci N, Cantiello HF. Bundles of brain microtubules generate electrical oscillations. Sci Rep. 2018:8:11899.

34. Bohrmann J, Dorn A, Sander K, Gutzeit H. The extracellular electrical current pattern and its variability in vitellogenic Drosophila follicles. J Cell Sci. 1986; 81:189-206.

35. Bohrmann J, Huebner E, Sander K, Gutzeit H. Intracellular electrical potential measurements in Drosophila follicles. J Cell Sci. 1986;81:207-21.

36. Bohrmann J. Potassium uptake into Drosophila ovarian follicles: relevance to physiological and developmental processes. J Insect Physiol. 1991;37:93746.

37. Bohrmann J, Heinrich U-R. Localisation of potassium pumps in Drosophila ovarian follicles. Zygote. 1994;2:189-99.

38. Bohrmann J, Braun B. Na, K-ATPase and V-ATPase in ovarian follicles of Drosophila melanogaster. Biol Cell. 1999;91:85-98.

39. Munley SM, Kinzeler S, Lizzano R, Woodruff RI. Fractional contribution of major ions to the membrane potential of Drosophila melanogaster oocytes. Arch Insect Biochem Physiol. 2009;70:230-43.

40. Mahowald AP, Kambysellis MP. Oogenesis. In: Ashburner M, Wright TRF, editors. Genetics and biology of Drosophila. New York: Academic Press; 1980. p. 141-224.

41. González-Reyes A, St Johnston D. Patterning of the follicle cell epithelium along the anterior-posterior axis during Drosophila oogenesis. Development. 1998;125:2837-46

42. Wu X, Tanwar PS, Raftery LA. Drosophila follicle cells: morphogenesis in an eggshell. Semin Cell Dev Biol. 2008;19:271-82.

43. Cavaliere V, Bernardi F, Romani P, Duchi S, Gargiulo G. Building up the Drosophila eggshell: first of all the eggshell genes must be transcribed. Dev Dyn. 2008;237:2061-72.

44. Ray RP, Schüpbach T. Intercellular signaling and the polarization of body axes during Drosophila oogenesis. Genes Dev. 1996;10:1711-23.

45. Riechmann V, Ephrussi A. Axis formation during Drosophila oogenesis. Curr Opin Genet Dev. 2001;11:374-83.

46. Roth S, Lynch JA. Symmetry breaking during Drosophila oogenesis. Cold Spring Harb Perspect Biol. 2009; 1:a001891.

47. Gutzeit $\mathrm{HO}$. The microfilament pattern in the somatic follicle cells of midvitellogenic ovarian follicles of Drosophila. Eur J Cell Biol. 1990;53:349-56.

48. Gutzeit HO, Eberhardt W, Gratwohl E. Laminin and basement membraneassociated microfilaments in wild-type and mutant Drosophila ovarian follicles. J Cell Sci. 1991;100:781-8.

49. Viktorinová I, König T, Schlichting K, Dahmann C. The cadherin Fat2 is required for planar cell polarity in the Drosophila ovary. Development. 2009; 136:4123-32.

50. Gates J. Drosophila egg chamber elongation: insights into how tissues and organs are shaped. Fly. 2012;6:213-27.

51. Viktorinová I, Dahmann C. Microtubule polarity predicts direction of egg chamber rotation in Drosophila. Curr Biol. 2013;23:1472-7.

52. Horne-Badovinac $S$. The Drosophila egg chamber - a new spin on how tissues elongate. Integr Comp Biol. 2014:54:667-76.

53. Viktorinová I, Henry I, Tomancak P. Epithelial rotation is preceded by planar symmetry breaking of actomyosin and protects epithelial tissue from cell deformations. PLoS Genet. 2017;13:e1007107.

54. Duhart JC, Parsons TT, Raftery LA. The repertoire of epithelial morphogenesis on display: progressive elaboration of Drosophila egg structure. Mech Dev. 2017;148:18-39.

55. Osterfield M, Berg CA, Shvartsman SY. Epithelial patterning, morphogenesis, and evolution: Drosophila eggshell as a model. Dev Cell. 2017;41:337-48

56. Tuszyński JA, Portet S, Dixon JM, Luxford C, Cantiello HF. Ionic wave propagation along actin filaments. Biophys J. 2004;86:1890-903.

57. Robb JA. Maintenance of imaginal discs of Drosophila melanogaster in chemically defined media. J Cell Biol. 1969;41:876-85.

58. Bohrmann J. In vitro culture of Drosophila ovarian follicles: the influence of different media on development, RNA synthesis, protein synthesis and potassium uptake. Roux Arch Dev Biol. 1991;199:315-26.

\section{Publisher's Note}

Springer Nature remains neutral with regard to jurisdictional claims in published maps and institutional affiliations.

\section{Ready to submit your research? Choose BMC and benefit from:}

- fast, convenient online submission

- thorough peer review by experienced researchers in your field

- rapid publication on acceptance

- support for research data, including large and complex data types

- gold Open Access which fosters wider collaboration and increased citations

- maximum visibility for your research: over $100 \mathrm{M}$ website views per year

At BMC, research is always in progress.

Learn more biomedcentral.com/submissions 